\title{
A Note on Spelling and Translation
}

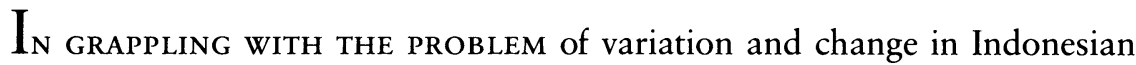
spellings I have opted for simplicity and consistency, while trying to maintain historical accuracy. I have spelled the names of people and institutions consistently throughout the book, generally using the simpler modern spellings rather than the older ones: $u$, not $o e ; j$, not $d j ; y$, not $j$; and $c$, not $t j$. The only exceptions to this rule are quotations from other sources and citations of authors and titles that use the old spellings. I have also retained the old spellings of the names of people who are best known by, or continue to use, those spellings.

The arcane vocabulary of Indonesian politics presents special translation problems, for the literal meanings of the terms are often either uninformative or misleading. To minimize confusion I have sometimes provided a gloss as well as a literal translation. All of the translations from Indonesian and Dutch sources are my own unless I have indicated otherwise. 